\title{
Exploration of potential objective and subjective daily indicators of sleep health in normal sleepers
}

This article was published in the following Dove Press journal:

Nature and Science of Sleep

\section{Sarah F Allen' \\ Greg J Elder' \\ Laura F Longstaff' \\ Zoe M Gotts ${ }^{2}$ \\ Rachel Sharman ${ }^{3}$ \\ Umair Akram ${ }^{4}$ \\ Jason G Ellis'}

'Department of Psychology, Faculty of Health and Life Sciences, Northumbria University, Newcastle upon Tyne, UK; ${ }^{2}$ Institute of Neuroscience, Campus for Ageing and Vitality, Newcastle University, Newcastle upon Tyne, UK; ${ }^{3}$ Sleep and Circadian Neuroscience Institute, Nuffield Department of Clinical Neuroscience, University of Oxford, Oxford, UK; ${ }^{4}$ Department of Psychology, Sociology and Politics, Sheffield Hallam University, Sheffield, UK
Correspondence: Jason G Ellis Department of Psychology, Faculty of Health and Life Sciences, Northumbria University, Newcastle upon Tyne NEI 8ST, UK

Tel +44 I9I 227 308I

Email jason.ellis@northumbria.ac.uk
Purpose: While the concept of "sleep health" has only recently been defined, how it relates to both subjective and objective sleep parameters is yet to be determined. The current study aimed to identify potential indicators of poorer sleep health, from subjective and objective daily sleep characteristics, in normal sleepers.

Participants and methods: Eighty-three individuals aged 18-65 years with no history of sleep disorders, chronic physical or psychiatric illnesses, or substance misuse were recruited from the North of England. Secondary analysis of a series of standardized studies, which included psychometrics, actigraphy, and an in-lab polysomnography (PSG) component, was undertaken. Questions from several psychometric sleep scales were combined to create an aggregate measure of sleep health status. Subjective sleep continuity was assessed by 2 -week sleep diary. Objective measures comprised two continuous weeks of actigraphy and two nights of in-lab PSG.

Results: Significant negative correlations were evident between sleep health scores and both diary-derived subjective sleep latency (SL; diary) and actigraphy-derived SL (actigraphy). This was reflected by independent samples $t$-test between high and low sleep health groups. No relationships between sleep health and PSG parameters were observed. Regression analyses indicated sleep latencies from both the sleep diary and actigraphy as significant predictors, explaining $28.2 \%$ of the variance in sleep health.

Conclusion: Perceived increases in SL appear to be a primary indicator of declining sleep health in normal sleepers. The majority of objective sleep parameters, including gross PSG sleep parameters, appear not to be sensitive to sleep health status in normal sleepers. Future research is needed to understand the physical and psychological correlates of sleep health in larger samples. Keywords: sleep health, PSG, actigraphy, normal sleepers

\section{Introduction}

In recent years, there has been a burgeoning interest in the relationship between sleep and health. ${ }^{1,2}$ Sleep is not only essential for the optimal performance of physical, ${ }^{3,4}$ cognitive, and emotional processes ${ }^{5}$ but also a vital biological determinant of everyday health and well-being. ${ }^{6}$ That said, to date sleep research has mainly focused on disturbed and/or disordered sleep, and the benefits of good healthy sleep, or "sleep health," have largely been overlooked. ${ }^{7,8}$ Furthermore, individual sleep characteristics do not occur in isolation, can be assessed across multiple dimensions of "sleep health," and are not specific to any particular sleep disorder. ${ }^{9}$

Although the research agenda in the area of sleep disorders has been invaluable in increasing our knowledge of the assessment, diagnosis, and management of sleep disorders, this focus tends to limit the understanding of sleep from a broader public 
health perspective. Additionally, while recent epidemiological and population health studies and research on the pathophysiology of sleep disorders, such as insomnia, ${ }^{10,11}$ continue to increase our understanding of vulnerability to sleep disorders and have provided insights into the consequences of poor sleep on health outcomes, the focus has still largely been on "abnormal." One potential reason for this focus on poor sleep and the limited research agenda on sleep health, to date, is that only recently have guidelines on what is considered "normal" or "typical" sleep, at least in terms of sleep duration and sleep quality, been outlined. ${ }^{12,13}$ As such, an understanding of what constitutes good sleep health has remained problematic.

Prior to the publication of the guidelines on "normal sleep" quantity and quality, Buysse ${ }^{7}$ outlined the first formal definition of "sleep health." He suggested that although sleep health could be viewed, in line with the medical model of health and illness, ${ }^{14}$ as an absence of disease, it was more likely to be a multidimensional construct and that one should focus on the positive attributes of sleep as opposed to sleep disturbance/disorder. To that end, and based upon his review of the relationships between specific aspects of the sleep experience and health outcomes, Buysse ${ }^{7}$ suggested that sleep health should encompass five subjective domains - an assessment of good or poor sleep (satisfaction), the ability to maintain attentive wakefulness (alertness), placement of sleep in the 24-hour day (timing), ease of falling asleep and staying asleep (efficiency), and total amount of sleep obtained per 24 hours (duration). To accompany his definition, Buysse ${ }^{7}$ developed a single scale to assess sleep health. The SATED scale comprises five questions pertaining to his definition (sleep Satisfaction, Alertness during waking, Timing of sleep, sleep Efficiency, and Duration of sleep). ${ }^{7}$ Further, he suggested that other elements of sleep, albeit more difficult to quantify, including an individual's ability to sleep when under threat or challenge (adaptability) and regularity in sleep schedules (variability), might be considered in more comprehensive assessments of sleep health.

More recently, the National Sleep Foundation (NSF) developed and psychometrically tested their own measure of sleep health - the "Sleep Health Index" (SHI) ${ }^{8}$ for use in large-scale studies. Employing a task force of experts, they developed an initial scale of 28 items that reduced, through factor analysis, into the final SHI, which consists of 12 items along three dimensions - sleep quality (six items), sleep duration (three items), and disordered sleep (three items). Within the sleep quality dimension, perceived sleep quality, feelings of restedness, difficulties falling or staying asleep, the negative impact from a lack of sleep, and unintentional dozing are included. For sleep duration, self-reported hours slept are benchmarked against the NSF's age-specific guidelines on sleep need in addition to measures of sleep deficit and sleep variability (workdays vs non-work days). Finally, the disordered sleep dimension asks about use of sleep medication, consultation with a health care professional about sleep, and whether the individual has been told by a health care professional that he or she has a sleep disorder.

While there are clearly overlaps between both scales, there are also some distinct differences. Similarities exist between the SATED's satisfaction, alertness, and efficiency dimensions and the SHI's sleep quality dimension, and between the SATED's duration and timing dimensions and the SHI's items measuring sleep duration. However, the SATED does not include a measure of disordered sleep or a specific measure of sleep deficit, whereas the SHI does not include adaptability.

Given that the research area of sleep health is relatively new, and the implications of sleep health are yet to be fully investigated, an important next step may be to determine whether there are any specific daily sleep indicators of the concept. If these indicators are identified, then changes in these sleep parameters are likely to signify a change in sleep health status which may facilitate early, or even preventative, interventions. This in itself has the potential to facilitate a public health agenda on sleep health, as knowing what the first signs of threat to an individuals' sleep health are may be an invaluable focus for a broad educational initiative.

The aim of the present study was to explore how retrospective assessment of sleep health, combining both recommendations from Buysse ${ }^{7}$ and the $\mathrm{SHI},{ }^{8}$ may be associated with particular subjective and objective daily sleep characteristics in a sample of normal sleepers. This will not only highlight how particular aspects of daily sleep may warrant further attention in relation to deterioration of sleep health but will also expand our knowledge of the utility of subjective sleep health measures in individuals free of sleep disorders. It was hypothesized that subjective sleep variables would be the best indicators of sleep health based upon the assertion that the definition of sleep health by Buysse ${ }^{7}$ and both the SATED and $\mathrm{SHI}^{8}$ rely on subjective reports.

\section{Methods}

\section{Participants}

Participants were 83 normal sleepers aged 18-65 years recruited for a series of studies in the North of the UK. The inclusion criteria for "normal sleeper" were as follows: 1) not meeting criteria for any sleep disorder, 2) defining themselves 
as a "normal" sleeper, 3) not seeing a health care professional regarding their sleep, and 4) not taking a medication (either prescribed or over the counter) for their sleep. The exclusion criteria were as follows: 1) a chronic illness (psychological or physical - including substance use disorders), 2) a previous head injury, and 3) not being able or willing to travel to the sleep center for overnight assessments. Demographic information for the full sample is displayed in Table 1.

\section{Procedure}

Participants were recruited through a series of media campaigns throughout the North of the UK. The media campaigns featured posters displayed in community settings (eg, community centers, libraries) and adverts in local newspapers. In each case, the campaign asked individuals, irrespective of whether they had a sleep problem or not, to contact the research team by telephone for more information. If a participant called, they were provided an overview of the study and once consent had been given they were screened for sleep disorders. If eligible and willing to participate, all participants were invited to attend the sleep laboratory for standardized baseline procedures. At this meeting, participants provided written informed consent and completed a face-to-face clinical interview to confirm that the participant met eligibility criteria. If eligible, participants were provided 2 weeks of sleep diaries, an actigraph, and a battery of psychometric assessments to complete at home. Additionally, an appointment was made for the participant to attend the sleep laboratory approximately 14 days later for an in-laboratory sleep assessment. At that appointment, participants returned the psychometric battery, the sleep diary, and the actigraph.

On each night of the study visit, participants arrived at the sleep laboratory at $8 \mathrm{pm}$ for study preparation (eg, electrode placement, bio-calibrations). Participants were instructed to

Table I Demographic characteristics

\begin{tabular}{|c|c|c|c|c|c|}
\hline & & $\mathbf{n}$ & $\%$ & $\begin{array}{l}\text { Low sleep health } \\
\text { group }\end{array}$ & $\begin{array}{l}\text { High sleep health } \\
\text { group }\end{array}$ \\
\hline & Age, M (SD) & $27.16(10.33)$ & & $26.60(10.71)$ & $27.71(10.34)$ \\
\hline \multirow[t]{2}{*}{ Gender } & Males & 41 & 49.41 & 18 & 21 \\
\hline & Females & 42 & 50.59 & 20 & 21 \\
\hline \multirow[t]{7}{*}{ Ethnicity } & White British & 64 & 77.06 & 30 & 33 \\
\hline & White Irish & 1 & 1.20 & I & 0 \\
\hline & White Scottish & 7 & 8.43 & 2 & 5 \\
\hline & Other White & 2 & 2.41 & 0 & 2 \\
\hline & Asian & 4 & 4.82 & 3 & I \\
\hline & Chinese & 2 & 2.41 & 1 & 1 \\
\hline & Undisclosed & 3 & 3.61 & I & 0 \\
\hline \multirow[t]{6}{*}{ Marital status } & Single & 64 & 77.06 & 33 & 30 \\
\hline & Married & 9 & 10.84 & 2 & 5 \\
\hline & Live-in partner & 5 & 6.02 & I & 4 \\
\hline & Divorced & 2 & 2.41 & I & I \\
\hline & Separated & I & 1.20 & 0 & I \\
\hline & Undisclosed & 2 & 2.41 & I & I \\
\hline Employment & Full time & 31 & 37.32 & 12 & 19 \\
\hline \multirow[t]{6}{*}{ status } & Part time & 2 & 2.41 & 0 & I \\
\hline & Unemployed & I & 1.20 & 0 & I \\
\hline & Retired & I & 1.20 & 0 & I \\
\hline & Student & 44 & 52.98 & 24 & 19 \\
\hline & Others & 2 & 2.41 & 1 & I \\
\hline & Undisclosed & 2 & 2.41 & I & 0 \\
\hline \multirow[t]{9}{*}{ Education } & Pre-bachelor & 15 & 18.06 & 4 & II \\
\hline & Bachelor & 38 & 45.75 & 19 & 19 \\
\hline & Master's & 10 & 12.04 & 6 & 4 \\
\hline & $\mathrm{PhD}$ & I & 1.20 & 0 & I \\
\hline & Diploma & 3 & 3.61 & 2 & I \\
\hline & College & 2 & 2.41 & 0 & I \\
\hline & MD & 2 & 2.41 & 0 & I \\
\hline & Others & 9 & 10.84 & 6 & 3 \\
\hline & Undisclosed & 3 & 3.61 & I & 0 \\
\hline Total & $\mathrm{N}=$ & 83 & & 38 & 42 \\
\hline
\end{tabular}


refrain from consuming alcohol, drugs, excessive caffeine, and nicotine before arrival. Bedtime was determined by the time reported in sleep diaries. Total recording period was for $\geq 8$ hours for all participants, although time out of bed in the morning was recorded and ambulatory PSG was employed if participants left the bedroom. All participants completed a sleep diary upon awakening. After electrodes were removed, participants left the laboratory and continued with their day. For all in-lab visits, taxis were provided, if necessary. All laboratory visits were undertaken on weekdays to limit the effect of altered sleep schedules on PSG readings. Participants were compensated $£ 80$ or $£ 150$ for their time depending upon the length of the study after baseline procedures (eg, two-night protocol vs three-night protocol).

The protocol for each study received ethical approval from the institutional ethics committees at Northumbria University and the University of Glasgow and conformed to the Declaration of Helsinki's ethical principles.

\section{Measures}

\section{Sleep health scores}

In order to address the dimensions of sleep health, as suggested by both Buysse ${ }^{7}$ and Knutson et al, ${ }^{8}$ a measure of sleep health was created using items from the Pittsburgh Sleep Quality Index, ${ }^{15}$ Insomnia Severity Index, ${ }^{16}$ Morningness-Eveningness Questionnaire, ${ }^{17}$ Ford Insomnia Response to Stress Test (FIRST), ${ }^{18}$ and Sleep Preoccupation Scale. ${ }^{19}$ Each question required respondents to consider their sleep and sleep-related behaviors, thoughts, and feelings over the previous month. An overview of each question and how they match with the SHI and SATED items is given in Table S1. For the purpose of the current study, the items used were grouped into the dimensions of Quality and Duration as proposed by the $\mathrm{SHI},{ }^{8}$ in addition to a dimension of Adaptability as recommended by Buysse. ${ }^{7}$ Considering that participants had been screened and excluded for having a sleep disorder and that a diagnosis of a sleep disorder and/or use of sleep medication were exclusion criteria in the present study, the dimension of "disordered sleep" as included in the SHI was not included in the present conceptualization of sleep health.

Sleep quality - Sleep quality was measured using five items conceptually similar to those included in the $\mathrm{SHI}^{8}$ and $\mathrm{SATED}^{7}$ - perceived sleep quality, sleep satisfaction, negative impact of lack of sleep, sleep efficiency (SE), and unintentional dozing during the day. The range of scores for this dimension was $0-18$, with higher scores indicating better sleep quality.

Sleep duration - Sleep duration was measured using three items - number of hours slept benchmarked against the NSF's age-appropriate criteria, sleep deficit, and sleep variability. The range of scores for this dimension was $0-8$, with higher scores indicating a good sleep duration.

Adaptability - Adaptability was measured using two items, combined, from the FIRST, which asked about the likelihood of daytime or evening stress impacting on sleep. The range of scores for this dimension was 0-6, with higher scores indicating better adaptability. This dimension was added in light of Buysse's ${ }^{7}$ recommendations that adaptability may be an important characteristic of sleep health.

\section{Subjective sleep}

The consensus sleep diary ${ }^{20}$ was used to create measures of subjective sleep continuity, including sleep latency (SL), wake after sleep onset (WASO), number of awakenings (NWAK), time in bed (TIB), total sleep time (TST), and SE $([T S T \div$ TIB] $\times 100)$ over the 2 -week period. Participants were instructed to complete the diary upon awakening each morning. Mean values were derived for each parameter for analyses.

\section{Objective sleep}

Polysomnography (PSG) - PSG data were derived from the second night (baseline) recording in the sleep laboratory. This was recorded on a 33-channel SomnoScreen plus (S-Med, Birmingham, UK). The first night of PSG was used as a screening/adaptation night and comprised an extended electroencephalogram (EEG) montage (including C3-A2; C4-A1), submental and anterior tibialis electromyograms (EMG), bilateral electrooculogram (EOG), heart rate, thoracic and abdominal respiratory effort, airflow (by nasal-oral thermocouple and nasal thermistor), and oxygen saturation via finger pulse oximetry. The second night (used for analyses) was a reduced montage and comprised the same EEGs, EMG (submental only), EOG, and heart rate measurements. Percentages of each sleep stage (Wake, N1, N2, SWS, and REM) in addition to measures of SL, WASO, NWAK, TST, TIB, and SE were derived for the present analysis. All PSGs were scored by a Registered Polysomnographic Technologistqualified technician using American Academy of Sleep Medicine guidelines; ${ }^{21}$ and $50 \%$ of those were also scored by a researcher (JGE) to confirm reliability.

Actigraphy - The Actiwatch (Actiwatch 7; CamnTech, Cambridge, UK) measured movement at 1 minute epochs. Sleep parameters were computed via computerized algorithms using the software "Actiwatch Activity \& Sleep Analysis 7" (CamnTech). Subjective "bed time" and "wakeup time," as determined by the sleep diary, were entered to produce a 
"Sleep Summary Report," from which mean values of sleep continuity variables (ie, SL, WASO, NWAK, TIB, TST, and $\mathrm{SE}$ ) were extracted. For the purposes of the present study, Fragmentation Index (FI) from the actigraph data was also included.

\section{Treatment of data}

Sleep health was considered as both a continuous and a categorical variable. Sleep health scores were calculated from the questions identified (Table S1) to provide a continuous sleep health score for each participant (range 0-32). Mean values for each diary and actigraphy parameter were used in analyses. Pearson's correlation analyses were initially undertaken between the sleep health scores and the diary, actigraphy, and PSG outcomes (Table S2), and multicollinearity was assumed if correlations between the predictor variables were above $r=0.7$. Taking an exploratory approach, hierarchical multiple regression analyses (enter method) were then undertaken with sleep health as the outcome and related sleep parameters as predictors. For categorical analyses, high and low sleep health scorers were distinguished via median split. Group differences were assessed using independent samples $t$-test. A significance level of $P<0.05$ was used for all analyses.

Missing data were replaced by mean substitution when $<5 \%$ of responses were missing; otherwise, case-wise deletion was implemented. Full sleep diary data were missing for one participant, and TST and SE (diary) data were missing for another individual. Actigraphy data were missing for five participants and FI was available for only 58 participants. PSG data were available for all 83 participants, with the exception of "percentage time awake" in the data of five individuals.

\section{Results}

The mean score on the measure of sleep health was 27.13 $(\mathrm{SD}=3.31)$ with a range from 17 to 32 . There were no significant differences between sex on sleep health scores ( $t[81]=-.367, P=0.715$ ) and the correlation between sleep health scores and age was $r=0.101$. Individuals with good and poor sleep health were then split by the median (three individuals scoring on the median were removed) for categorical analyses. This resulted in 38 individuals in the low sleep health group and 42 in the high sleep health group. Demographic data for both groups are listed in Table 1.

\section{Subjective sleep continuity}

With respect to subjective sleep continuity parameters (sleep diary), a significant negative correlation $(r=-0.467, P<0.001)$ between sleep health and SL was demonstrated. A significant between-group difference was also only observed for subjective SL $(t[77]=2.744, P=0.008)$, with individuals measuring high on sleep health demonstrating shorter SL $(M=13.44$, $\mathrm{SD}=7.52)$ than low sleep health scores $(\mathrm{M}=19.79, \mathrm{SD}=12.61)$. All other correlations and between-group comparisons were nonsignificant (Table S2).

\section{Objective sleep}

$P S G$-No significant correlations were evident between sleep health scores and any sleep continuity, or sleep architecture parameters as determined by PSG. Independent samples $t$-test also indicated no significant between-group differences on these measures (Table S2).

Actigraphy - With regard to sleep continuity as assessed by actigraphy, a significant negative correlation was observed between sleep health and SL $(r=-0.375, P<0.001)$. No other significant relationships were evident. Independent samples $t$-test $(t[74]=2.805, P=0.006)$ indicated that the high sleep health group $(\mathrm{M}=29.61, \mathrm{SD}=26.55)$ reported significantly shorter SLs than the low sleep health group ( $M=49.59$, $\mathrm{SD}=35.33$ ). No other significant between-group differences were observed (Table S2).

\section{Regression model}

Considering the correlation coefficients between actigraphy and sleep diary measures of TST ( $r=0.83)$, actigraphy measures of TST and TIB $(r=0.80)$ and actigraphy measures of NWAK and WASO $(r=0.78)$ were above 0.70 . The actigraphic variables for TST and NWAK were not included in the regression model due to multicollinearity. The correlation between SL diary and SL actigraphy measures did not indicate collinearity $(r=0.28)$. At Step 1 , the model including age and sex as predictors of sleep health was not significantly better than the grand mean $(F[2,74]=0.789, P=0.458)$. The addition of SL (diary) to the model was, however, significant at Step 2 $(F[3,73]=7.394, P<0.001)$, with subjective SL (diary) predicting an additional $21.2 \%$ of the variance. The final model was also significant at Step $3(F[4,72]=7.077, P<0.001)$, with objective SL (actigraphy) predicting a further $4.9 \%$ of the variance in sleep health. The final model explained $28.2 \%$ of the variance in sleep health. Both subjective SL (diary) and objective SL (actigraphy) were significant predictors in the final model, and SL (diary) was the strongest predictor ( $\beta=0.351)$ (Table 2).

\section{Discussion}

The aim of the current study was to assess how sleep health may be related to subjective and objective sleep parameters in 
Table 2 Multiple regression analyses demonstrating the prediction of sleep health scores by age, sex, subjective SL (diary), and objective SL (actigraphy)

\begin{tabular}{|c|c|c|c|c|c|c|c|}
\hline \multicolumn{2}{|c|}{ Predictors } & \multirow{2}{*}{$\begin{array}{l}\text { B } \\
0.034\end{array}$} & \multirow{2}{*}{$\begin{array}{l}\text { SE B } \\
0.036\end{array}$} & \multirow{2}{*}{$\begin{array}{l}\boldsymbol{\beta} \\
0.109\end{array}$} & \multirow{2}{*}{$\begin{array}{l}T \\
0.945\end{array}$} & \multirow{2}{*}{$\begin{array}{l}P \\
0.348\end{array}$} & \multirow{2}{*}{$\begin{array}{l}R^{2}\left(\Delta R^{2}\right) \\
0.02 I\end{array}$} \\
\hline Step I & Age & & & & & & \\
\hline & Sex & $0.58 I$ & 0.758 & 0.088 & 0.767 & 0.446 & \\
\hline \multirow[t]{3}{*}{ Step 2} & Age & 0.036 & 0.032 & 0.115 & 1.123 & 0.265 & \\
\hline & Sex & 0.400 & 0.677 & $0.06 \mathrm{I}$ & 0.592 & 0.556 & \\
\hline & SL (diary) & -0.141 & 0.031 & -0.461 & -4.494 & $<0.00 I^{* *}$ & $233^{* *}\left(0.212^{* *}\right)$ \\
\hline \multirow[t]{4}{*}{ Step 3} & Age & 0.014 & 0.033 & 0.045 & 0.428 & 0.670 & \\
\hline & Sex & 0.053 & 0.678 & 0.008 & 0.079 & 0.937 & \\
\hline & SL (diary) & -0.121 & 0.032 & -0.394 & -3.765 & $<0.00 I^{* *}$ & \\
\hline & SL (actigraphy) & -0.026 & 0.012 & -0.250 & -2.221 & $0.030^{*}$ & $0.282 * *(0.049 *)$ \\
\hline
\end{tabular}

Notes: $* * P<0.001, * P<0.05$.

Abbreviations: SL, sleep latency; $B$, unstandardized beta; SE $B$, standard error of unstandardized beta; $\beta$, standardized beta; $R^{2}, \mathrm{R}$-squared; $\Delta R^{2}$, R-squared change.

normal sleepers, as determined by sleep diaries, actigraphy, and PSG. This is the first study to the authors' knowledge to examine these daily sleep characteristics in relation to an aggregate measure of sleep health. Associations were observed between sleep health status and SL, both from sleep diaries and actigraphy. Furthermore, the current study provided evidence that these two factors in combination could, to a certain extent, predict sleep health. These findings, combined with the findings from the $t$-tests, ultimately indicate that shorter SL plays a role in determining good sleep health. The SL findings potentially demonstrate evidence of consistency between subjective (diary) and objective (actigraphy) measures.

However, as actigraphy also requires aspects of selfreport, it is suggested that the determining factors of sleep health are likely to be largely subjective. Nevertheless, given that actigraphy is largely informed by sleep diaries, it is not surprising that these measures of SL were both related to sleep health. Individual's perception of their sleep in the determination of what is "healthy sleep" is therefore fundamental. In a similar way, there is an abundance of evidence that self-perceptions of health and well-being can influence physical and mental health outcomes. ${ }^{22}$

The current findings have implications with regard to a public health agenda on sleep. The knowledge that SL could potentially be an indicator of sleep health provides opportunities in this context. Primarily, it provides a simple quantifiable marker of an individual's overall sleep health, which could easily be framed in a public health campaign, as an empowerment tool (eg, knowing your SL score), a call to action (eg, observing a negative change in sleep health status), or even a sense of improvement following a change in behavior (eg, observing a positive change in sleep health status). That said, as the sample consisted of mainly European Caucasian younger adults, these results should be viewed carefully with regard to generalizability to all ages and cultures.

Interestingly, relationships were not observed between sleep health scores and any PSG markers, suggesting that sleep health status may not be easily inferred by PSG sleep parameters. The incongruity between subjective sleep and PSG measures in the current study is not uncommon, as other studies have also demonstrated links between self-reported sleep, but not objective sleep, and aspects of health. ${ }^{23}$ However, as the PSG data were extracted from only a single night, it is possible that sleep health cannot be defined or identified simply within only a single night's sleep and that more nights of recording are necessary. Additionally, as the data from the second night were used, this may represent recovery from the first night familiarization, further supporting this premise.

The current study benefits from the use of numerous assessments of sleep to identify the most significant indicators of sleep health. Furthermore, the benchmarking of sleep duration and sleep quality against age-appropriate norms, from the NSF, provides a clearer understanding of these factors in context. Nevertheless, certain limitations must also be acknowledged. The current study largely comprised selfmonitoring, which is often an atypical period for participants and, therefore, may have affected the variability of the sleep variables recorded. Although 2 weeks of actigraphy and sleep diary assessment should have overcome these stability issues, some parameters, such as PSG, often take longer to stabilize. ${ }^{24}$ Calculation of sleep timing stability in future studies may be beneficial to assess this issue. Furthermore, actigraphy has questionable reliability and validity, given the difficulty in distinguishing quiet wakefulness from sleep. ${ }^{25} \mathrm{As}$ such, the findings, specifically with respect to the PSG and 
actigraphy, should be viewed with a degree of caution. Additionally, as previously mentioned, given the initial selection criteria of healthy, normal sleepers, this study lacks the ability to generalize findings to a clinical (eg, insomniacs) or unbiased community sample. Therefore, it would be of value to verify these findings in individuals with insomnia and other sleep disorders (ie, those with obviously poor sleep health), in comparison to normal sleepers. Furthermore, as "normal sleep" status was gaged subjectively, the possibility that some participants (particularly in the low-sleep health group) may have had undiagnosed sleep disorders must be considered.

Finally, the use of several different items from several psychometric scales to determine sleep health status could be considered a limitation. However, aggregate multidimensional measures of sleep health have previously been implemented successfully in this way. ${ }^{9}$ Furthermore, the questions used to assess sleep health combined aspects of both the $\mathrm{SHI}^{8}$ and $\mathrm{SATED}^{7}$ scales to provide the most comprehensive overview of what is considered sleep health, albeit retrospectively self-reported. Additionally, all the items were taken from well-validated scales. Future research should, however, also seek to replicate these findings using the SATED, the SHI, or indeed both with more heterogeneous populations.

\section{Conclusion}

Although research indicates sleep health is a subjective, multidimensional construct, it has been demonstrated that perceptions of SL may be an indicator of sleep health in individuals free from sleep disorders. It is suggested that this particular daily sleep characteristic may therefore warrant extra consideration in normal sleepers (ie, individuals who consider themselves free of sleep disorders) to maintain their sleep health. No evidence has been provided to suggest that PSG sleep parameters can reliably predict declines in sleep health in normal sleepers. However, longer PSG studies would be required to confirm this. The current study also exemplifies the utility of this aggregate sleep health measure as a quick and easy way to assess the concept in a representative group of normal sleepers. Nevertheless, future studies are warranted with larger samples to determine the factor structure of the construct, its psychometric properties, and other physical and psychological correlates of sleep health.

\section{Acknowledgments}

The Sleep Council provided financial support for the preparation of this article. Part of the data collection for this study was funded by a grant received from the ESRC for the Sleep GAPS project. This paper was presented at the $32 \mathrm{nd}$ annual meeting of the Associated Professional Sleep Societies as a poster presentation with interim findings. The Poster's abstract was published in "Basic and Translational Sleep Science” in SLEEP, Volume 41, Abstract Supplement, 2018. https://doi.org/10.1093/sleep/zsy061.325.

\section{Disclosure}

The authors report no conflicts of interest in this work.

\section{References}

1. Czeisler CA. Duration, timing and quality of sleep are each vital for health, performance and safety. Sleep Health. 2015;1(1):5-8.

2. Irwin MR, Olmstead R, Carroll JE. Sleep Disturbance, Sleep Duration, and Inflammation: A Systematic Review and Meta-Analysis of Cohort Studies and Experimental Sleep Deprivation. Biol Psychiatry. 2016;80(1):40-52.

3. Markov D, Goldman M. Normal sleep and circadian rhythms: neurobiologic mechanisms underlying sleep and wakefulness. Psychiatr Clin North Am. 2006;29(4):841-853.

4. Spiegel K, Leproult R, van Cauter E. Impact of sleep debt on metabolic and endocrine function. Lancet. 1999;354(9188):1435-1439.

5. Maquet P. The role of sleep in learning and memory. Science. 2001;294(5544):1048-1052.

6. Prendergast KB, Schofield GM, Mackay LM. Associations between lifestyle behaviours and optimal wellbeing in a diverse sample of New Zealand adults. BMC Public Health. 2015;16(1):62.

7. Buysse DJ. Sleep health: can we define it? Does it matter? Sleep. 2014;37(1):9-17.

8. Knutson KL, Phelan J, Paskow MJ, et al. The National Sleep Foundation's sleep health index. Sleep Health. 2017;3(4):234-240.

9. Furihata R, Hall MH, Stone KL, et al. Study of Osteoporotic Fractures (SOF) Research Group. An Aggregate Measure of Sleep Health Is Associated With Prevalent and Incident Clinically Significant Depression Symptoms Among Community-Dwelling Older Women. Sleep. 2016;40(3):1-11.

10. Morin CM, Bélanger L, Leblanc M, et al. The natural history of insomnia: a population-based 3-year longitudinal study. Arch Intern Med. 2009; 169(5):447-453.

11. Ellis JG, Perlis ML, Neale LF, Espie CA, Bastien CH. The natural history of insomnia: focus on prevalence and incidence of acute insomnia $J$ Psychiatr Res. 2012;46(10):1278-1285.

12. Ohayon M, Wickwire EM, Hirshkowitz M, et al. National Sleep Foundation's sleep quality recommendations: first report. Sleep Health. 2017;3(1):6-19.

13. Hirshkowitz M, Whiton K, Albert SM, et al. National Sleep Foundation's sleep time duration recommendations: methodology and results summary. Sleep Health. 2015;1(1):40-43.

14. Engel GL. The need for a new medical model: a challenge for biomedicine. Science. 1977;196(4286):129-136.

15. Buysse DJ, Reynolds CF, Monk TH, Berman SR, Kupfer DJ. The Pittsburgh Sleep Quality Index: a new instrument for psychiatric practice and research. Psychiatry Res. 1989;28(2):193-213.

16. Morin CM, Savard J, Ouellet MC, Daley M. Insomnia. Hoboken, NJ: John Wiley \& Sons, Inc; 1993.

17. Horne JA, Ostberg O. A self-assessment questionnaire to determine morningness-eveningness in human circadian rhythms. Int J Chronobiol. 1976;4(2):97-110.

18. Drake C, Richardson G, Roehrs T, Scofield H, Roth T. Vulnerability to stress-related sleep disturbance and hyperarousal. Sleep. 2004;27(2):285-291.

19. Ellis J, Mitchell K, Hogh H. Sleep preoccupation in poor sleepers: psychometric properties of the Sleep Preoccupation Scale. J Psychosom Res. 2007;63(6):579-585. 
20. Carney CE, Buysse DJ, Ancoli-Israel S, et al. The consensus sleep diary: standardizing prospective sleep self-monitoring. Sleep. 2012;35(2):287-302.

21. Iber C, Ancoli-Israel S, Chesson A, and Quan SF for the American Academy of Sleep Medicine. The AASM Manual for the Scoring of Sleep and Associated Events: Rules, Terminology and Technical Specifications. 1st. Ed. Westchester, Illinois: American Academy of Sleep Medicine; 2007.

22. Diener E, Chan MY. Happy People Live Longer: Subjective Well-Being Contributes to Health and Longevity. Appl Psychol. 2011;3(1):1-43.
23. Bei B, Milgrom J, Ericksen J, Trinder J. Subjective perception of sleep, but not its objective quality, is associated with immediate postpartum mood disturbances in healthy women. Sleep. 2010;33(4):531-538.

24. Wohlgemuth WK, Edinger JD, Fins AI, Sullivan RJ. How many nights are enough? The short-term stability of sleep parameters in elderly insomniacs and normal sleepers. Psychophysiology. 1999;36(2):233-244.

25. Sadeh A. The role and validity of actigraphy in sleep medicine: an update. Sleep Med Rev. 2011;15(4):259-267. 


\section{Supplementary materials}

Table SI Sleep health dimensions, questions, and scoring

\begin{tabular}{|c|c|c|c|c|}
\hline $\begin{array}{l}\text { Sleep health } \\
\text { index dimension }\end{array}$ & $\begin{array}{l}\text { Sleep health } \\
\text { components }\end{array}$ & Question & Scoring & $\begin{array}{l}\text { Corresponding item from } \\
\text { SHI/SATED }\end{array}$ \\
\hline \multirow[t]{5}{*}{$\begin{array}{l}\text { Sleep quality } \\
\text { (SHI component) }\end{array}$} & I. Sleep quality & $\begin{array}{l}\text { How would you rate your sleep quality } \\
\text { overall? }\end{array}$ & $\begin{array}{l}\text { Very good }=3 \\
\text { Fairly good }=2 \\
\text { Fairly bad }=1 \\
\text { Very bad }=0\end{array}$ & $\begin{array}{l}\text { How many days have you } \\
\text { woke up feeling well-rested? } \\
(\mathrm{SHI})\end{array}$ \\
\hline & 2. Sleep satisfaction & $\begin{array}{l}\text { How satisfied are you with your current } \\
\text { sleep pattern? }\end{array}$ & $\begin{array}{l}\text { Very satisfied }=4 \\
\text { Satisfied }=3 \\
\text { Moderately satisfied }=2 \\
\text { Dissatisfied }=1 \\
\text { Very dissatisfied }=0\end{array}$ & $\begin{array}{l}\text { Are you satisfied with your } \\
\text { sleep? (SATED) }\end{array}$ \\
\hline & 3. Negative impact & $\begin{array}{l}\text { I cannot perform my daily tasks as well } \\
\text { when I have had a bad night's sleep...? }\end{array}$ & $\begin{array}{l}\text { Always }=6 \\
\text { Almost all of the time }=5 \\
\text { Quite a lot }=4 \\
\text { Sometimes }=3 \\
\text { Very infrequently }=2 \\
\text { Hardly ever }=1 \\
\text { Never }=0\end{array}$ & $\begin{array}{l}\text { How many days did poor or } \\
\text { insufficient sleep significantly } \\
\text { impact your daily activities, } \\
\text { like your work performance, } \\
\text { socializing, exercising, or other } \\
\text { typical activities? (SHI) }\end{array}$ \\
\hline & 4. Sleep efficiency & $\begin{array}{l}\text { i) How many hours of actual sleep } \\
\text { did you get at night? ii) When have } \\
\text { you usually gone to bed at night? iii) } \\
\text { When have you usually gotten up in } \\
\text { the morning? (number of hours slept/ } \\
\text { number of hours spent in bed } \times 100 \text { ) } \\
\text { (scored using NSF guidelines by age } \\
\text { group) }\end{array}$ & $\begin{array}{l}\text { I) } 18-25 \text { years old } \\
>85 \%=2 \\
65-84 \%=1 \\
<65 \%=0 \\
\text { 2) } 26-64 \text { years old } \\
>85 \%=2 \\
75-84 \%=1 \\
<75 \%=0\end{array}$ & $\begin{array}{l}\text { Do you spend less than } 30 \\
\text { minutes awake at night? } \\
\text { (including the time it takes to } \\
\text { fall asleep and awakenings from } \\
\text { sleep) (SATED) }\end{array}$ \\
\hline & $\begin{array}{l}\text { 5. Unintentional } \\
\text { dozing }\end{array}$ & $\begin{array}{l}\text { How often have you had trouble staying } \\
\text { awake while driving, eating meals, or } \\
\text { engaging in social activities? }\end{array}$ & $\begin{array}{l}\text { No problem at all }=3 \\
\text { Only a very slight problem }=2 \\
\text { Somewhat of a problem }=1 \\
\text { A very big problem }=0\end{array}$ & $\begin{array}{l}\text { How many days did you fall } \\
\text { asleep without intending to, such } \\
\text { as dozing off in front of the TV } \\
\text { or in any other situation? (SHI) }\end{array}$ \\
\hline \multirow[t]{3}{*}{$\begin{array}{l}\text { Sleep duration } \\
\text { (SHI component) }\end{array}$} & 6. Sleep duration & $\begin{array}{l}\text { How many hours of actual sleep did you } \\
\text { get at night? } \\
\text { (scored using NSF guidelines by age } \\
\text { group) }\end{array}$ & $\begin{array}{l}\text { I) } 18-25 \text { years old } \\
7-9 \text { hours }=2 \\
6 \text { or } 10-11 \text { hours }=1 \\
<6 \text { or }>11 \text { hours }=0 \\
2 \text { ) } 26-64 \text { years old } \\
7-9 \text { hours }=2 \\
6 \text { or } 10 \text { hours }=1 \\
<6 \text { or }>10 \text { hours }=0\end{array}$ & $\begin{array}{l}\text { Do you sleep between } 6 \text { and } 8 \\
\text { hours per day? (SATED) }\end{array}$ \\
\hline & 7. Sleep deficit & $\begin{array}{l}\text { i) Approximately what time would } \\
\text { you get up if you were entirely free to } \\
\text { plan your day? ii) Approximately what } \\
\text { time would you go to bed if you were } \\
\text { entirely free to plan your evening? (ideal } \\
\text { duration and difference with actual sleep } \\
\text { duration calculated) }\end{array}$ & $\begin{array}{l}<1 \text { hour }=3 \\
1-2 \text { hours }=2 \\
2-3 \text { hours }=1 \\
3-4 \text { hours }=0\end{array}$ & $\begin{array}{l}\text { How many hours of sleep do } \\
\text { you need to be well-rested and } \\
\text { feel your best? (SHI) }\end{array}$ \\
\hline & 8. Sleep variability & $\begin{array}{l}\text { If you had no commitments the next } \\
\text { day, what time would you go to bed } \\
\text { compared to your usual bedtime? }\end{array}$ & $\begin{array}{l}\text { Seldom } / \text { never later }=3 \\
<I \text { hour later }=2 \\
I-2 \text { hours later }=1 \\
2+\text { hours later }=0\end{array}$ & $\begin{array}{l}\text { What about on non-work days } \\
\text { or weekends - what time did } \\
\text { you most often go to bed on } \\
\text { those days? (SHI) }\end{array}$ \\
\hline \multirow[t]{2}{*}{$\begin{array}{l}\text { Adaptability } \\
\text { (additional } \\
\text { component) }\end{array}$} & $\begin{array}{l}\text { 9. Sleep } \\
\text { adaptability } \\
\text { (daytime reactivity) }\end{array}$ & $\begin{array}{l}\text { How likely is it for you to have difficulty } \\
\text { sleeping after a stressful experience } \\
\text { during the day? }\end{array}$ & $\begin{array}{l}\text { Not likely }=3 \\
\text { Somewhat likely }=2 \\
\text { Moderately likely }=1 \\
\text { Very likely }=0\end{array}$ & $\begin{array}{l}\text { Sleep adaptability questions } \\
\text { were included in light of } \\
\text { recommendations by Buysse }\end{array}$ \\
\hline & $\begin{array}{l}\text { 10. Sleep } \\
\text { adaptability } \\
\text { (evening reactivity) }\end{array}$ & $\begin{array}{l}\text { How likely is it for you to have difficulty } \\
\text { sleeping after a stressful experience in } \\
\text { the evening? }\end{array}$ & $\begin{array}{l}\text { Not likely=3 } \\
\text { Somewhat likely=2 } \\
\text { Moderately likely=1 } \\
\text { Very likely }=0\end{array}$ & \\
\hline
\end{tabular}

Abbreviations: NSF, National Sleep Foundation; SATED, satisfaction, alertness, timing, efficiency, and duration; SHI, Sleep Health Index. 
Table S2 Analyses for the measures of subjective sleep (diary) and objective sleep (PSG and actigraphy): I) Pearson's correlations with continuous sleep health scores and 2) between-group (high vs low $\mathrm{SH}$ ) differences

\begin{tabular}{|c|c|c|c|c|c|c|c|c|c|c|c|}
\hline & \multicolumn{3}{|c|}{ Correlations } & \multicolumn{5}{|c|}{ Between groups } & \multirow{2}{*}{$\begin{array}{l}95 \% \\
\text { LCL }\end{array}$} & \multirow{2}{*}{$\begin{array}{l}95 \% \\
\text { UCL }\end{array}$} & \multirow[t]{2}{*}{ D } \\
\hline & $R$ & $P$ & $M( \pm S D)$ & $\begin{array}{l}\text { Low SH } \\
\text { scorers } \\
M( \pm S D) \\
n=38\end{array}$ & $\begin{array}{l}\text { High SH } \\
\text { scorers } \\
M( \pm S D) \\
n=42\end{array}$ & $T$ & $d f$ & $P$ & & & \\
\hline \multicolumn{12}{|l|}{ Subjective sleep (diary) } \\
\hline SL (minutes) & -0.467 & $<0.00 I^{* *}$ & $16.66(10.61)$ & $19.79(12.61)$ & I3.44 (7.52) & 2.744 & 77 & $0.008^{*}$ & 1.74 & 10.97 & 0.63 \\
\hline WASO (minutes) & -0.203 & 0.068 & $9.55(10.70)$ & $10.97(11.05)$ & $8.77(10.59)$ & 0.902 & 77 & 0.370 & -2.65 & 7.05 & 0.21 \\
\hline TIB (minutes) & 0.087 & 0.435 & $522.25(59.83)$ & $517.99(66.29)$ & $522.96(54.92)$ & -0.364 & 77 & 0.717 & -32.17 & 22.22 & 0.08 \\
\hline TST (minutes) & 0.105 & 0.353 & 463.21 (48.39) & 459.84 (55.33) & $464.47(4 I .86)$ & -0.419 & 76 & 0.677 & -26.69 & 17.42 & 0.09 \\
\hline NWAK & 0.051 & 0.650 & $0.94(0.75)$ & $0.92(0.77)$ & $1.02(0.73)$ & -0.583 & 77 & 0.562 & -4.35 & 0.24 & 0.13 \\
\hline$\% \mathrm{SE}$ & 0.185 & 0.098 & $87.09(8.73)$ & $85.61(9.10)$ & $88.86(8.39)$ & -1.637 & 76 & 0.106 & -7.19 & 0.70 & 0.38 \\
\hline \multicolumn{12}{|c|}{ PSG sleep continuity parameters } \\
\hline SL (minutes) & 0.063 & 0.569 & $19.50(17.18)$ & $20.12(20.39)$ & $18.24(14.09)$ & 0.483 & 78 & 0.631 & -5.86 & 9.61 & 0.11 \\
\hline NWAK & 0.166 & 0.147 & $9.72(7.86)$ & $8.11(6.23)$ & $10.83(8.40)$ & $\mathrm{I} .577$ & 73 & 0.336 & -6.14 & 0.72 & 0.37 \\
\hline WASO (minutes) & 0.056 & 0.614 & $22.63(35.40)$ & $27.29(46.09)$ & $18.60(23.34)$ & 1.079 & 78 & 0.284 & -7.35 & 24.72 & 0.24 \\
\hline TST (minutes) & -0.155 & 0.161 & $4 \mid 3.30(58.79)$ & $419.80(61.98)$ & $409.59(55.11)$ & 1.257 & 78 & 0.212 & -15.85 & 36.27 & 0.29 \\
\hline$\%$ SE & -0.017 & 0.877 & $90.5 \mathrm{I}(17.95)$ & $89.73(13.00)$ & $92.14(21.25)$ & -0.606 & 78 & 0.546 & -10.36 & 5.52 & 0.14 \\
\hline \multicolumn{12}{|c|}{ PSG sleep architecture parameters } \\
\hline Percentage wake & 0.187 & 0.102 & $20.84(34.7 I)$ & $12.47(24.37)$ & $26.79(38.96)$ & -1.889 & 73 & 0.063 & -14.32 & 7.58 & 0.44 \\
\hline Percentage Stage I & 0.041 & 0.712 & $26.62(24.91)$ & $24.13(27.69)$ & $29.32(21.01)$ & -0.948 & 78 & 0.346 & -16.06 & 5.70 & 0.21 \\
\hline Percentage Stage 2 & -0.049 & 0.659 & $39.26(19.18)$ & $41.65(18.67)$ & $36.62(19.51)$ & 1.177 & 78 & 0.243 & -3.48 & 13.56 & 0.27 \\
\hline Percentage slow wave sleep & -0.188 & 0.089 & $14.84(6.91)$ & $15.75(6.50)$ & $14.30(7.4 \mid)$ & 0.924 & 78 & 0.359 & -1.67 & 4.56 & 0.21 \\
\hline Percentage REM & 0.108 & 0.331 & $25.32(14.57)$ & $23.74(13.77)$ & $26.95(15.66)$ & -0.969 & 78 & 0.336 & -9.80 & 3.39 & 0.22 \\
\hline \multicolumn{12}{|l|}{ Actigraphy sleep parameters } \\
\hline SL (minutes) & -0.375 & $<0.00 I^{* *}$ & 39.18 (32.37) & $49.59(35.33)$ & $29.6 I(26.55)$ & 2.805 & 74 & $0.006 *$ & 5.79 & 34.18 & 0.65 \\
\hline NWAK & -0.003 & 0.981 & $27.72(8.77)$ & $28.49(10.43)$ & $26.83(6.93)$ & 0.704 & 74 & 0.484 & -4.69 & 3.14 & 0.16 \\
\hline WASO (minutes) & -0.100 & 0.383 & $55.49(39.54)$ & $61.20(40.49)$ & $51.66(38.70)$ & 1.050 & 74 & 0.297 & -8.56 & 27.65 & 0.24 \\
\hline TST (minutes) & 0.040 & 0.729 & $402.99(60.04)$ & $393.16(67.51)$ & $409.92(52.93)$ & -1.211 & 74 & 0.230 & -44.35 & 10.83 & 0.28 \\
\hline$\%$ SE & 0.168 & 0.141 & 80.41 (.8.29) & $78.38(8.83)$ & $82.00(7.60)$ & -1.920 & 74 & 0.059 & -7.37 & 0.14 & 0.45 \\
\hline $\mathrm{FI}$ & -0.081 & 0.548 & $35.51(14.94)$ & $37.38(16.91)$ & $33.93(12.69)$ & -0.858 & 54 & 0.675 & $-4.6 I$ & 11.50 & 0.20 \\
\hline
\end{tabular}

Notes: $* * P<0.001, * P<0.05$

Abbreviations: Fl, Fragmentation Index; LCL, lower confidence limit; NWAK, number of awakenings; PSG, polysomnography; SE, sleep efficiency; SH, sleep health; SL, sleep latency; TIB, time in bed; TST, total sleep time; UCL, upper confidence limit; WASO, wake after sleep onset.

Nature and Science of Sleep

\section{Publish your work in this journal}

Nature and Science of Sleep is an international, peer-reviewed, open access journal covering all aspects of sleep science and sleep medicine, including the neurophysiology and functions of sleep, the genetics of sleep, sleep and society, biological rhythms, dreaming, sleep disorders and therapy, and strategies to optimize healthy sleep. The manuscript

\section{Dovepress}

management system is completely online and includes a very quick and fair peer-review system, which is all easy to use. Visit http://www dovepress.com/testimonials.php to read real quotes from published authors. 